Tendências em Matemática Aplicada e Computacional, 3, No. 1 (2002), 81-90.

(C) Uma Publicação da Sociedade Brasileira de Matemática Aplicada e Computacional.

\title{
Vibrações Forçadas em uma Viga Fixa-Livre Sujeita a um Torque e Movimento no Apoio
}

R.D. COPETTI ${ }^{1}$ Promec, UFRGS e Departamento de Matemática, CCNE, UFSM, 97105-900 Santa Maria, RS, Brasil.

J.R. CLAEYSSEN ${ }^{2}$, Instituto de Matemática - Promec, UFRGS, 90001-000 Porto Alegre, RS, Brasil.

Resumo. Este trabalho tem como objetivo analisar os efeitos da força externa e distúrbios de fronteira sobre a resposta de um sistema modelado segundo a teoria para vigas uniformes tipo Euler-Bernoulli. A resposta do sistema é determinada em termos da resposta impulso e das respostas devido aos distúrbios de fronteira. Este processo é realizado no próprio espaço físico do problema, sem a necessidade do uso da formulação do espaço de estado, através de uma ánalise modal direta na equação de segunda ordem.

\section{Introdução}

O propósito deste trabalho é analisar os efeitos de uma força externa e distúrbios nas extremidades de uma figa fixa-livre descrita pelo modelo de Euler-Bernoulli e sujeita a forças de amortecimento viscoso e material. Para tanto, é utilizada a análise modal diretamente no espaço físico [2], [3], preservando características próprias, como simetria e positividade. Isto não é o caso com a formulação do espaço de estado comumente encontrada na literatura [4], [7].

Sistemas distribuídos, nos quais os parâmetros são dependentes do domínio espacial, são descritos por problemas de contorno, consistindo de uma ou várias equações diferenciais parciais que devem ser satisfeitas sobre um domínio $\Omega$ para um número apropriado de condições de contorno e dados iniciais.

A resposta transiente é caracterizada em termos da resposta impulso e de suas derivadas. A análise modal é utilizada no cálculo da resposta impulso e a inclusão dos distúrbios é realizada com o auxílio da função de Green espacial.

São apresentadas simulações numéricas para os modos de vibração, resposta impulso, efeitos da força externa, movimento na base, torque e variação dos parâmetros de amortecimento.

\footnotetext{
${ }^{1}$ rmaira@oslo.ccne.ufsm.br

2 julio@mat.ufrgs.br
} 


\section{Modelo Físico e Modelo Matemático}

Considere uma viga fixa-livre conforme Figura 1 sujeita a uma excitação $w_{b}(t)$ na sua extremidade esquerda, um torque $\tau_{b}(t)$ na extremidade direita e uma força externa $f(t, x)$ concentrada em um ponto qualquer ao longo do comprimento da viga. Assumimos distúrbios iniciais nulos.

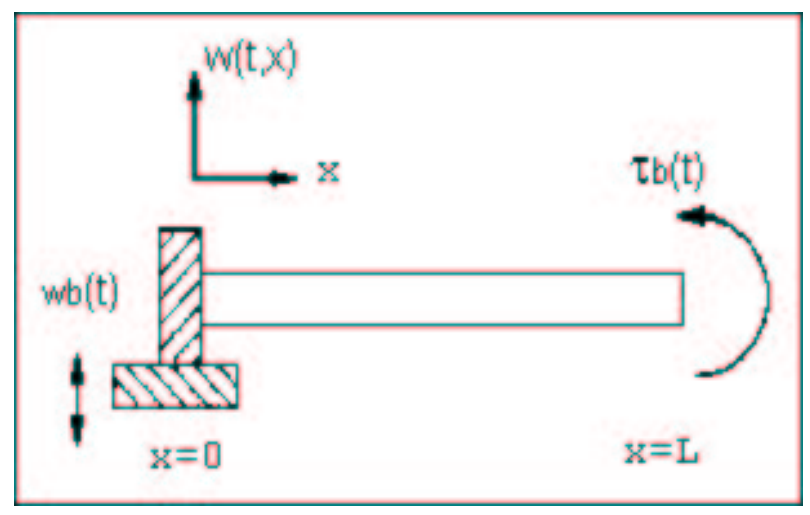

Figura 1: Viga sujeita a distúrbios de fronteira

O modelo matemático é obtido considerando-se uma viga uniforme do tipo Euler-Bernoulli. Deste modo, a resposta $w(t, x)$ é solução do problema nãohomogêneo com condições de contorno não-homogêneas dado por:

$$
\begin{aligned}
& M w_{t t}(t, x)+D w_{t}(t, x)+K w(t, x)=f(t, x), \\
& w(0, x)=0, \quad \frac{\partial}{\partial t} w(0, x)=0, \\
& w(t, 0)=w_{b}(t), \quad \frac{\partial w}{\partial x}(t, 0)=0, \\
& \frac{\partial}{\partial t}\left(\beta \frac{\partial^{2} w(t, L)}{\partial x^{2}}\right)+E I \frac{\partial^{2} w(t, L)}{\partial x^{2}}=\tau_{b}(t), \\
& \frac{\partial}{\partial t}\left(\beta \frac{\partial^{3} w(t, L)}{\partial x^{3}}\right)+\frac{\partial}{\partial x}\left(E I \frac{\partial^{2} w(t, L)}{\partial x^{2}}\right)=0,
\end{aligned}
$$

onde $M, D$ e $K$ são operadores diferenciais espaciais definidos por

$$
M=\rho, \quad D=\alpha+\beta \frac{\partial^{4}}{\partial x^{4}} \quad \text { e } K=\frac{\partial^{2}}{\partial x^{2}}\left(E I \frac{\partial^{2}}{\partial x^{2}}\right)
$$

e as constantes

$$
\begin{array}{ll}
\rho: \text { densidade linear, } & E I: \text { rigidez da viga, } \\
\alpha: \text { atrito viscoso, } & \beta: \text { atrito material. }
\end{array}
$$

\section{Resposta Forçada através do Método Espectral}

Primeiro, obteremos a resposta para o problema não-homogêneo com condições de contorno homogêneas para depois, então, resolver o problema acima proposto. 
O problema homogêneo associado a (2.1) admite solução do tipo exponencial $w(t, x)=$ $e^{\lambda t} v(x)$ se $v(x)$ é solução do problema de contorno

$$
\left[\lambda^{2} M+\lambda D+K\right] v(x)=0, \quad x \in[0, L],
$$

onde $M, D$ e $K$ atuam sobre funções $v(x)$ tais que

$$
v(0)=0, \quad v^{\prime}(0)=0, \quad v^{\prime \prime}(L)=0, \quad v^{\prime \prime \prime}(L)=0 .
$$

Procura-se, então, solução da forma

$$
w(t, x)=\sum_{k=1}^{\infty} c_{k}(t) e^{\lambda_{k} t} v_{k}(x),
$$

que, substituido naquela equação, obtém-se, pelo método de variação de parâmetros,

$$
\sum_{k=1}^{2 n} \lambda_{k} e^{\lambda_{k} t} \dot{c}_{k}(t) M v_{k}(x)=f, \quad \sum_{k=1}^{2 n} e^{\lambda_{k} t} \dot{c}_{k}(t) v_{k}(x)=0 .
$$

Multiplicando-se, em (3.3), a primeira equação por $\lambda_{j} \bar{v}_{j}^{T}$ e a segunda por $\bar{v}_{j}^{T} K$, e integrando-se de 0 a L, obtemos:

$$
\sum_{k=1}^{2 n} e^{\lambda_{k} t} \dot{c}_{k}(t)\left\langle v_{j}, \lambda_{k} \lambda_{j} M v_{k}(x)-K v_{k}(x)\right\rangle=\lambda_{j}\left\langle v_{j}, f\right\rangle,
$$

onde

$$
\langle u, v\rangle=\int_{0}^{L} u^{*} v d x
$$

e $\left(^{*}\right)$ indica a conjugação complexa.

No caso da viga ser uniforme, o módulo de Young, E, e o momento de inércia, I, são constantes. Observa-se, então, que D é do tipo Rayleigh. Assim, tem-se que (3.1) pode ser escrito como

$$
K v=\omega^{2} M v,
$$

onde

$$
\omega^{2}=-\frac{\lambda^{2}+a \lambda}{\lambda b+1}, \quad a=\frac{\alpha}{\rho} \quad \text { e } \quad b=\frac{\beta}{E I},
$$

o qual corresponde ao problema de contorno de autovalor

$$
\begin{array}{ll}
v^{i v}-\beta^{4} v=0, & \beta^{4}=\frac{\omega^{2} \rho}{E I}, \\
v(0)=0, & v^{\prime}(0)=0 \\
v^{\prime \prime}(L)=0, & v^{\prime \prime \prime}(L)=0
\end{array}
$$

que possui um conjunto ortogonal de autofunções reais, [6], isto é,

$$
\left\langle v_{k}, M v_{j}\right\rangle=\delta_{k j} \quad \text { e } \quad\left\langle v_{k}, K v_{j}\right\rangle=\omega_{k}^{2} \delta_{k j} .
$$

Em particular,

$$
\lambda_{k}^{2}\left\langle v_{k}, M v_{k}\right\rangle-\left\langle v_{k}, K v_{k}\right\rangle=\lambda_{k}^{2}-\omega_{k}^{2} .
$$


Portanto, de (3.4), tem-se

$$
e^{\lambda_{k} t} \dot{c}_{k}(t) \gamma_{k}=\lambda_{k}\left\langle v_{k}, f\right\rangle ;
$$

substituindo o produto interno na equação acima e integrando no tempo de 0 a t, tem-se

$$
c_{k}(t)=\frac{\lambda_{k}}{\gamma_{k}} \int_{0}^{t} e^{-\lambda_{k} \tau} \int_{0}^{L} v_{k}(\xi) f(\xi, \tau) d \xi d \tau .
$$

Substituindo-se (3.6) em (3.2), obtemos a resposta do sistema (2.1) com condições de contorno homogêneas, na forma forçada compacta, em função dos autovalores e autovetores do problema de autovalor de segunda ordem associado (3.1) ou (3.5), isto é,

$$
w(t, x)=\int_{0}^{t} \int_{0}^{L} h(t-\tau, x, \xi) f(\tau, \xi) d \xi d \tau,
$$

onde, para $\lambda_{k}$ autovalor e $v_{k}$ o autovetor associado, tem-se

$$
h(t, x, \xi)=\sum_{k=1}^{\infty} \frac{\lambda_{k}}{\gamma_{k}} e^{\lambda_{k} t} v_{k}(x) v_{k}(\xi)
$$

$\operatorname{com} \gamma_{k}=\left\langle u_{k}, \lambda_{k}^{2} M u_{k}-K u_{k}\right\rangle \neq 0, \quad k=1,2, \cdots$.

Pode ser verificado que $h$ é solução do sistema distribuído amortecido,

$$
\begin{aligned}
& M h_{t t}(t, x, \xi)+D h_{t}(t, x, \xi)+K h(t, x, \xi)=\delta(t) \delta(x-\xi), \quad x, \xi \in \Omega, \quad t>0 \\
& \Gamma h(t, x, \xi)=0, \quad x \in \partial \Omega, \quad \xi \in \Omega, \\
& h(0, x, \xi)=0, \quad h_{t}(0, x, \xi)=0, \quad x \in \Omega, \quad \xi \in \Omega
\end{aligned}
$$

As Figuras 2 e 3 mostram um gráfico dos modos de vibração da viga, $w_{n}(t)=e^{\alpha_{n} t} \operatorname{sen} \beta_{n} t$, para $n=1 \ldots 4, \quad x=6 / 7$, onde os $\lambda_{n}=\alpha_{n}+i \beta_{n}$ são os autovalores do problema. Foram utilizados os seguintes parâmetros [7]:

\begin{tabular}{|l|l|l|l|}
\hline $\mathrm{E}=1$ & Módulo de Young & $\rho=16$ & Densidade linear \\
$\mathrm{L}=1$ & Comprimento da barra & $\alpha=1.6$ & Atrito viscoso \\
$\mathrm{I}=1$ & Inércia & $\beta=0.001$ & Atrito material \\
\hline
\end{tabular}

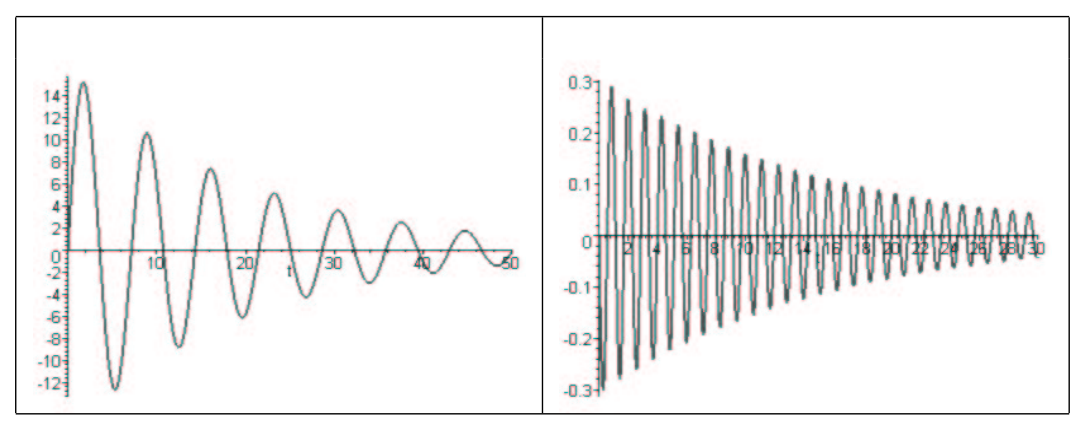

Figura 2: Modos de Vibração da viga para $n=1$ e $n=2$ 


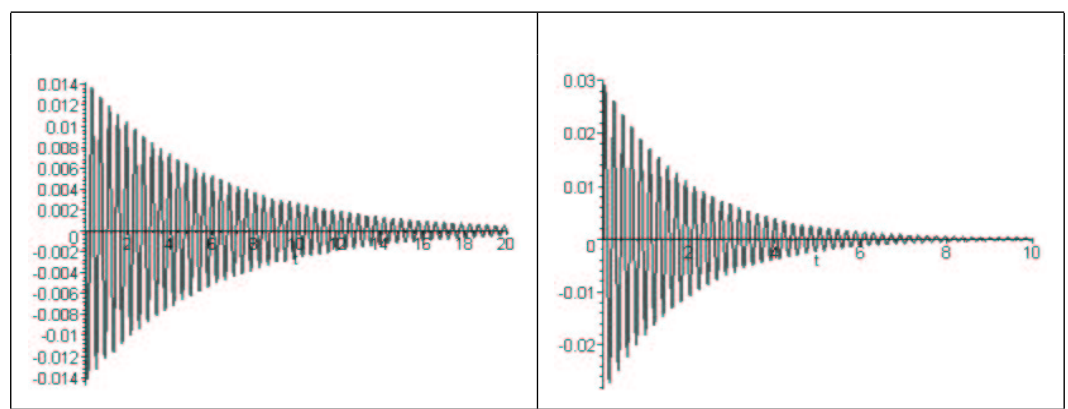

Figura 3: Modos de Vibração da viga para $n=3$ e $n=4$

A seguir mostrar-se-á um gráfico aproximado da função de Green da viga fixalivre.

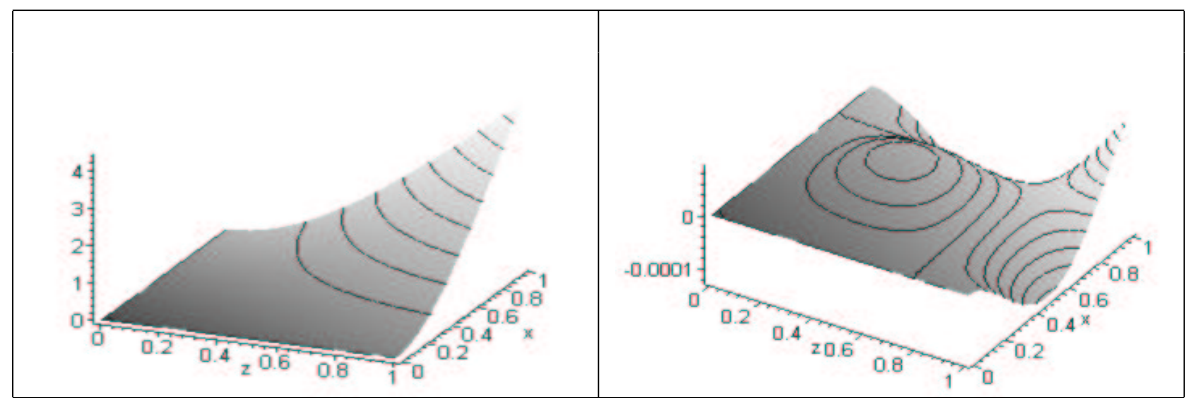

Figura 4: Linhas de contorno da Resposta Impulso da viga em t=2 e t=10.8

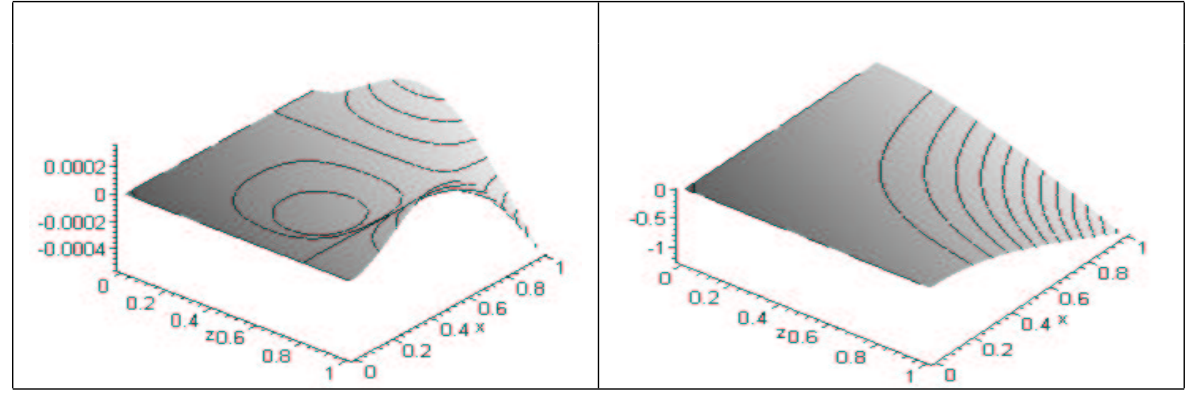

Figura 5: Linhas de contorno da Resposta Impulso da viga em t=17.9 e t=27 


\section{Problema Não-Homogêneo com Condições de Contorno Não-Homogêneas}

Obtida a solução para o problema não-homogêneo com condições de contorno homogêneas, considera-se o problema geral, ou seja, o problema não-homogêneo com condições de contorno não-homogêneas dado por (2.1).

Através da transformada de Laplace aplicada à equação (2.1), tem-se

$$
\begin{aligned}
\left(s^{2} \rho+s \alpha\right) W(s, x)+\frac{\partial^{2}}{\partial x^{2}}\left((s \beta+E I) \frac{\partial^{2}}{\partial x^{2}} W(s, x)\right) & =F(s, x), \\
W(s, 0)=W_{b}(s), & \frac{\partial W}{\partial x}(s, 0) \\
s \beta \frac{\partial^{2} W(s, L)}{\partial x^{2}}+E I \frac{\partial^{2} W(s, L)}{\partial x^{2}} & =\mathrm{T}_{b}(s), \\
s \beta \frac{\partial^{3} W(s, L)}{\partial x^{3}}+\frac{\partial}{\partial x}\left(E I \frac{\partial^{2} W(s, L)}{\partial x^{2}}\right) & =0,
\end{aligned}
$$

onde $W(s, x), F(s, x), W_{b}(s)$ e $\mathrm{T}_{b}(s)$ são as transformadas de Laplace de $w(t, x)$, $f(t, x), w_{b}(t)$ e $\tau_{b}(t)$, respectivamente.

Para se obter o deslocamento $w(t, x)$ em termos de uma carga arbitrária $F$, considera-se $H(s, x, \xi)$ como sendo função de Green espacial associada ao problema (4.2) [6], isto é, satisfazendo

$$
\begin{aligned}
\left(s^{2} \rho+s \alpha\right) H(s, x, \xi)+\frac{\partial^{2}}{\partial \xi^{2}}\left((s \beta+E I) \frac{\partial^{2}}{\partial \xi^{2}} H(s, x, \xi)\right) & =\delta(x-\xi), \quad x, \xi \in(0, L) ; \\
H(s, x, 0)=0, & \frac{\partial}{\partial \xi} H(s, x, 0) \\
(s \beta+E I)\left[\frac{\partial^{2}}{\partial \xi^{2}} H(s, x, \xi)\right]_{\xi=L} & =0 ; \quad x \in(0, L) ; \\
s \beta\left[\frac{\partial^{3}}{\partial \xi^{3}} H(s, x, \xi)\right]_{\xi=L}+\left[\frac{\partial}{\partial \xi} E I \frac{\partial^{2}}{\partial \xi^{2}} H(s, x, \xi)\right]_{\xi=L} & =0 .
\end{aligned}
$$

Multiplicando-se ambos os lados da primeira equação em $\left(4.2^{\circ}\right)$ à esquerda por $H(s, x, \xi)$ e integrando-se por partes de 0 a L, têm-se

$$
\begin{aligned}
& \mathrm{B}(W, G)+\int_{0}^{L} W(s, \xi)\left(s^{2} \rho+s \alpha\right) H(s, x, \xi) d \xi \\
& \quad+\int_{0}^{L} W(s, \xi) \frac{\partial^{2}}{\partial \xi^{2}}\left((s \beta+E I) \frac{\partial^{2}}{\partial \xi^{2}} H(s, x, \xi)\right) d \xi=\int_{0}^{L} H(s, x, \xi) F(s, \xi) d \xi
\end{aligned}
$$


onde

$$
\begin{aligned}
\mathrm{B}(W, G)= & s \beta\left[H(s, x, \xi) \frac{\partial^{3}}{\partial \xi^{3}} W(s, \xi)-\frac{\partial}{\partial \xi} H(s, x, \xi) \frac{\partial^{2}}{\partial \xi^{2}} W(s, \xi)\right. \\
& +\frac{\partial^{2}}{\partial \xi^{2}} H(s, x, \xi) \frac{\partial}{\partial \xi} W(s, \xi)-\frac{\partial^{3}}{\partial \xi^{3}} H(s, x, \xi) W(s, \xi) \\
& +H(s, x, \xi) \frac{\partial}{\partial \xi}\left(E I \frac{\partial^{2}}{\partial \xi^{2}} W(s, \xi)\right)-\frac{\partial}{\partial \xi} H(s, x, \xi)(E I) \frac{\partial^{2}}{\partial \xi^{2}} W(s, \xi) \\
& +\frac{\partial^{2}}{\partial \xi^{2}} H(s, x, \xi)(E I) \frac{\partial}{\partial \xi} W(s, \xi) \\
& \left.-\frac{\partial}{\partial \xi}\left(E I \frac{\partial^{2}}{\partial \xi^{2}} H(s, x, \xi)\right) w(s, \xi)\right]_{0}^{L} .
\end{aligned}
$$

Substituindo-se as condições de contorno para $W(s, x)$, dadas por (4.2), e para $H(s, x, \xi)$, dadas por (4.2), na equação (4.4), segue-se

$$
\begin{aligned}
\mathrm{B}(W, G)= & {\left[\frac{\partial}{\partial \xi}(s \beta+E I) \frac{\partial^{2}}{\partial \xi^{2}} H(s, x, \xi)\right]_{\xi=0} W_{b}(s) } \\
& -\left[\frac{\partial}{\partial \xi} H(s, x, \xi)\right]_{\xi=L} \mathrm{~T}_{b}(s) .
\end{aligned}
$$

Por outro lado, multiplicando-se a primeira equação em (4.2) à esquerda por $W(s, \xi)$, integrando-se de 0 a L e usando uma propriedade da função $\delta$, obtemos

$$
\int_{0}^{L} W(s, \xi)\left(\left(s^{2} \rho+s \alpha\right) H(s, x, \xi)+\frac{\partial^{2}}{\partial \xi^{2}}\left((s \beta+E I) \frac{\partial^{2}}{\partial \xi^{2}}\right) H(s, x, \xi)\right) d \xi=W(s, x)
$$

Substituindo-se (4.6) em (4.3), obtemos

$$
W(s, x)=-\mathrm{B}(W, G)+\int_{0}^{L} H(s, x, \xi) F(s, \xi) d \xi .
$$

De (4.5) segue que

$$
\begin{aligned}
W(s, x)=- & {\left[\frac{\partial}{\partial \xi}(s \beta+E I) \frac{\partial^{2}}{\partial \xi^{2}} H(s, x, \xi)\right]_{\xi=0} W_{b}(s) } \\
& +\left[\frac{\partial}{\partial \xi} H(s, x, \xi)\right]_{\xi=L} \mathrm{~T}_{b}(s)+\int_{0}^{L} H(s, x, \xi) F(s, \xi) d \xi
\end{aligned}
$$


Introduzindo

$$
\begin{aligned}
h_{1}(t, x, \xi) & =\mathcal{L}^{-1}\left[H_{1}(s)\right]=\mathcal{L}^{-1}-\left[\frac{\partial}{\partial \xi}(s \beta+E I) \frac{\partial^{2}}{\partial \xi^{2}} H(s, x, \xi)\right]_{\xi=0} \\
& =-\left[\frac{\partial}{\partial \xi}\left(\beta \frac{\partial}{\partial t}+E I\right) \frac{\partial^{2}}{\partial \xi^{2}} h(t, x, \xi)\right]_{\xi=0} \\
h_{2}(t, x, \xi) & =\mathcal{L}^{-1}\left[H_{2}(s)\right]=\mathcal{L}^{-1}\left[\frac{\partial}{\partial \xi} H(s, x, \xi)\right]_{\xi=L}=\left[\frac{\partial}{\partial \xi} h(t, x, \xi)\right]_{\xi=L} \\
h(t, x, \xi) & =\mathcal{L}^{-1}[H(s, x, \xi)]
\end{aligned}
$$

e aplicando transformada inversa de Laplace em (4.7), têm-se

$w(t, x)=\int_{0}^{t}\left(h_{1}(t-\tau, x, \xi) w_{b}(\tau)+h_{2}(t-\tau, x, \xi) \tau_{b}(\tau)+\int_{0}^{L} h(t-\tau, x, \xi) f(\tau, \xi) d \xi d \tau\right.$.

A equação (4.10) pode ser escrita na forma da integral de convolução como sendo

$$
w(t, x)=\int_{0}^{t} \mathrm{~h}(t-\tau, x, \xi) \mathbf{f}(\tau, x) d \tau,
$$

onde $\mathrm{h}$ denota o operador que atua sobre a entrada geral do sistema, isto é, dos distúrbios de fronteira e do termo forçante

$$
\mathbf{f}(\tau, x)=\left[\begin{array}{c}
w_{b}(\tau) \\
\tau_{b}(\tau) \\
f(\tau, x)
\end{array}\right]
$$

através de

$$
\mathrm{h}(t, x, \xi) \mathbf{f}(\tau, x)=\int_{0}^{L}\left[h_{1}(t, x, \xi) \delta(\xi) h_{2}(t, x, \xi) \delta(\xi) h(t, x, \xi)\right]\left[\begin{array}{c}
w_{b}(\tau) \\
\tau_{b}(\tau) \\
f(\tau, \xi)
\end{array}\right] d \xi
$$

Observe que para se determinar a resposta do sistema (2.1), dada por (4.11), é necessário determinar $\mathrm{h}(t, x, \xi)$. Porém, para tanto, por (4.12) torna-se necessário determinarmos $h_{1}(t, x, \xi), h_{2}(t, x, \xi)$ e $h(t, x, \xi)$. Mas, por (4.9), $h_{1}$ e $h_{2}$ são dadas em função de $h$. Logo, a resposta do sistema (2.1) fica determinada se encontrarmos a expressão para $h$, a função de Green ou resposta impulso associada a esse sistema.

Para entradas oscilatórias amortecidas, $e^{\alpha_{n} t} \operatorname{sen}\left(\beta_{n} t\right)$, perto de um autovalor do sistema, $\lambda_{n}=\alpha_{n}+i \beta_{n}$, como mostra a Figura 6 , e do tipo $A e^{\gamma t}$, como mostra a Figura 7 , na base $x=0$, torque na extremidade $x=L$ e uma carga concentrada em $x=a$, observou-se, nas simulações, que as respostas são semelhantes, diferindo apenas nas amplitudes. Nestas figuras, tem-se a primeira simulação com força externa ativada e movimento na base e torque nulos, na seguinte tem-se movimento 
na base ativado com força externa e torque nulos e na terceira tem-se força externa, movimento na base e torque ativados.

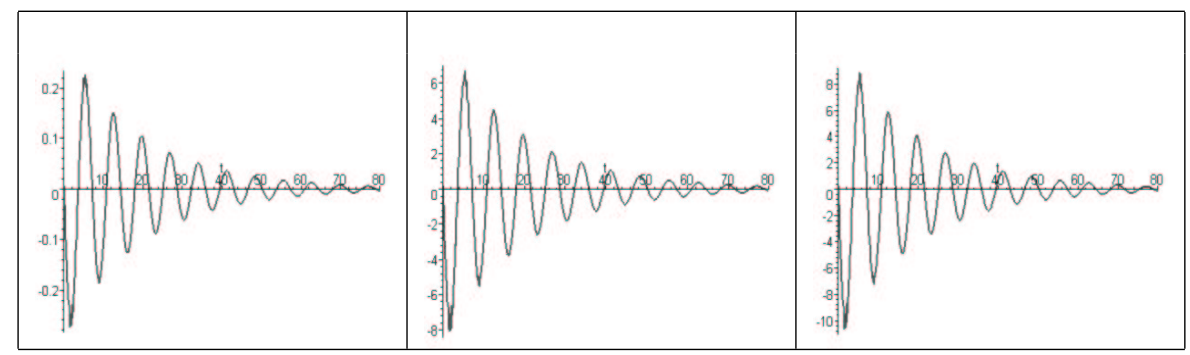

Figura 6: Resposta para viga fixa livre, para $\alpha_{3}=-0.16, \beta_{3}=15.42$ e $a=1 / 3$

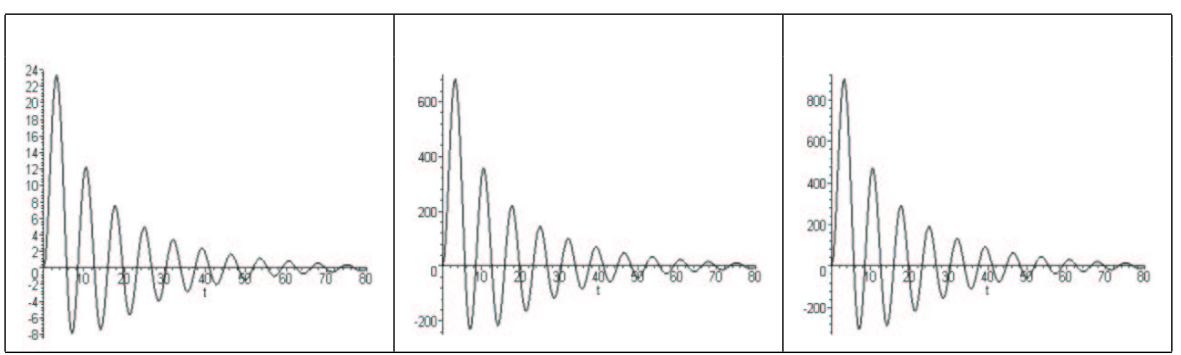

Figura 7: Resposta para viga fixa livre para $A=3.5, \gamma=-0.2$ e $a=1 / 3$

O efeito do movimento na base do tipo $A e^{\alpha_{n} t} \operatorname{sen} \beta_{n} t$, perto dos três primeiros autovalores $\lambda_{n}, n=1,2$ e 3, sobre a resposta do sistema é ilustrado na Figura(8). Foi observado que o efeito do torque, ainda que qualitativamente semelhante ao movimento na base, é de menor amplitude. Isto deve ocorrer uma vez que os modos de vibração em $h_{1}(t, x, \xi)$ possuem uma ponderação maior que os correspondentes em $h_{2}(t, x, \xi)$.

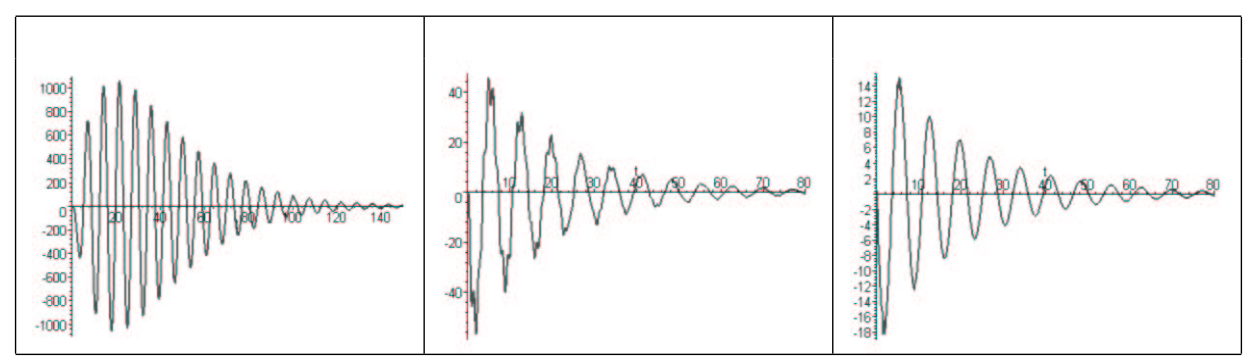

Figura 8: Resposta para viga fixa livre com variação no torque, para $\mathrm{n}=1, \mathrm{n}=2$ e $\mathrm{n}=3$

Nas simulações a seguir, foi observado o efeito do atrito viscoso $\alpha$ sobre a res- 
posta do sistema. As simulações foram feitas para uma força externa concentrada em $x=a$ do tipo $e^{\alpha_{n} t} \operatorname{sen} \beta_{n} t$, próximo do primeiro autovalor $(n=1)$, com torque e movimento na base nulos. Observou-se que a resposta mantém o comportamento, porém diminuindo amplitude conforme o atrito vai aumentando como era o esperado.

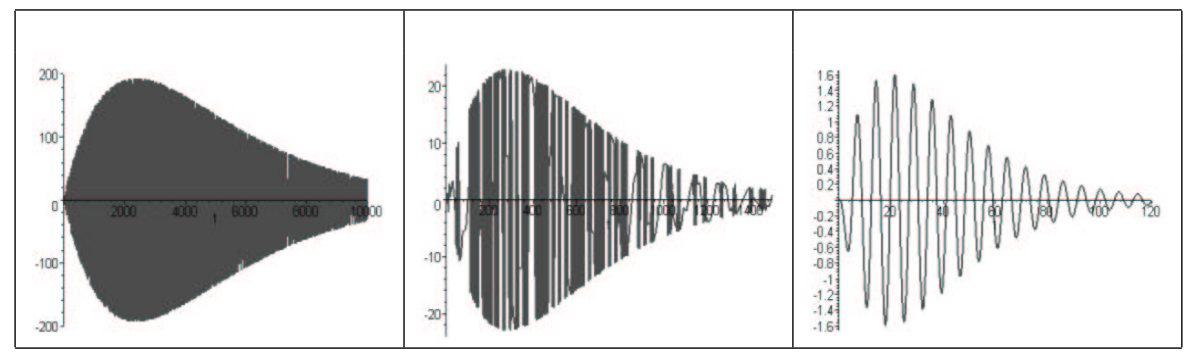

Figura 9: Resposta para viga fixa livre para $\alpha=0.001, \quad \alpha=0.1$ e $\alpha=1.6$

Abstract. The objective of this work is to analyze the effects of the external force and boundary disturbances on the response of a system modeled according to the theory for uniform beams type Euler-Bernoulli. The system response is obtained in terms of the impulse response and of the responses due to boundary disturbances. This process is accomplished in the own physical space of the problem, without need of the state space approach, through a direct modal analysis in the second order equation.

\section{Referências}

[1] A.G. Butkovskiy, "Structural Theory of Distributed Systems", John Wiley, New York, 1983.

[2] J.R. Claeyssen, G.C. Suazo, C.R. Jung, A direct approach to second-order matrix non-classical vibrating equations, Applied Numerical Mathematics, 30 (1999), 65-78.

[3] J.R. Claeyssen, L.D. Chiwiacowsky, G.C. Suazo, The impulse response in the symbolic computating of modes for beams and plates, Applied Numerical Mathematics, 40 (2002), 119-135.

[4] J.H. Ginsberg, "Mechanical and Structural Vibrations - Theory and Applications", John Wiley, New York, 2001.

[5] D. Inman, "Engineering Vibration", Prentice Hall, New Jersey, 1994.

[6] L. Meirovitch, "Principles and Techniques of Vibrations", Prentice-Hall, Inc., New Jersey, 1997.

[7] B. Yang, Closed-form transient response of distributed damped systems, Part 1: Modal analysis and Green's function formula, ASME Journal of Applied Mechanics, 63 (1996), 997-1003. 